\title{
SUFRIMIENTO HUMANO Y CONTEXTOS DE AJUSTE TERAPÉUTICO
}

\author{
HUMAN SUFFERING AND THERAPEUTICAL ADJUSTMENT CONTEXTS \\ FREDRIK LÚCAR VIDAL ${ }^{1}$ \\ Universidad Nacional Mayor de San Marcos, Perú \\ (Recibido el 18/08/2010, ACEPTAdo el 16/11/2010
}

\section{RESUMEN}

El artículo se basa en la relevancia de un planteamiento cultural de los problemas psicológicos contemporáneos. En ese sentido, se tratará de analizar la repercusión a nivel psicológico de algunos fenómenos sociales posmodernos tales como la ansiedad por la felicidad como objetivo personal (el rechazo de todo tipo de sufrimiento), el incremento desmesurado de las expectativas y la excesiva necesidad de control. No se niega ni subestima otros factores pero se incide en la importancia de estos. Asimismo, y en función al análisis esbozado, se señalará la necesidad -cada vez más actual- de la aplicación del enfoque conductual de tercera generación, como un camino fiable y con sustento apropiadamente averiguado dentro de la psicología, haciendo especial énfasis en la Terapia de Aceptación y Compromiso (TAC) como una de las psicoterapias más pertinentes para el manejo de dichos malestares.

Palabras clave: Sufrimiento, bienestar, felicidad, necesidad de control, terapia de aceptación y compromiso.

\begin{abstract}
The article is based on the relevance of having a cultural approach to psychological problems that occur nowadays. In that sense, we attempt to analyze what is the impact in a psychological level of some postmodern social phenomena such as anxiety for the happiness as a personal goal (rejection of all kinds of suffering), the huge increment of expectations and the excessive need for control. We do not deny nor underestimate other factors however we stress the importance of these. Likewise, according to the analysis proposed before, we will indicate the necessity in the implementation of Third Generation Behavior Therapy, as a reliable and also wel.researched path inside psychology, with special emphasis in Acceptance and Commitment Therapy (ACT), which is one of the most relevant psychotherapies for the treatment of these problems.
\end{abstract}

Keywords: Suffering, welfare, happiness, need for control, acceptance and commitment therapy.

1 Bachiller en Psicología de la Facultad de Psicología de la UNMSM. E-mail: flucarv@gmail.com 


\section{INTRODUCCIÓN}

Siguiendo la línea que propone la relevancia de un planteamiento cultural de los problemas psicológicos contemporáneos (Bautista \& Quiroga, 2005; Martín, 2006), veremos que los principales malestares psicológicos de hoy (ni hablar de los trastornos agudos y graves) deben su mantenimiento y empeoramiento a algunas cuestiones cardinales tales como la ansiedad por la felicidad, el incremento desmesurado de las expectativas, la excesiva necesidad de control, y en general, la búsqueda de un bienestar constante en nuestras vidas que trae consigo la negación del sufrimiento humano como parte consustancial a la propia existencia (sufrimiento que englobaría todo un conjunto de factores interrelacionados de la propia existencia humana, que podría decirse que han existido desde el inicio de la humanidad, y que ahora han llegado a valores críticos en cuanto a su repercusión a nivel psicológico en las personas). Se trata pues de la paradoja de vivir en un mundo, en sentido amplio, más cómodo, más seguro, más preciso, muchísimo menos doloroso, y encontrarnos abrumados por el sufrimiento generado, precisamente por ese mundo diseñado para buscar la felicidad y evitar el sufrimiento. Parecería que antes las personas no ansiaban demasiadas comodidades, y aunque no haya sido así, el hecho es que actualmente avivamos ardorosamente nuestro anhelo de bien(estar) para poder vivir.

Es así que en este artículo no se pretenderá lucir una crítica social rigurosa y extendida ni tampoco confundir los niveles de análisis. Se tratará, entonces, de ver la repercusión psicológica en las personas a partir de los algunos cambios sociales posmodernos, y de allí, siguiendo la perspectiva conductual de tercera generación, se presentará la idea de una sustitución de la "necesidad control" de nuestras vidas por un muy necesario "distanciamiento compresivo".

\section{EL SUFRIMIENTO HUMANO}

La vida se puede resumir en tres palabras: triste, ridícula y puerca; sin embargo, nosotros podemos derramar algo de regocijo en esa tristeza, algo de elevación en esa ridiculez y algo de limpieza en esa porquería.

Manuel González Prada

Obras, I, 370

Las palabras de González Prada pueden percibirse como drásticas y exageradas, pero llevan algo (o mucho) de sentido cuando nos detenemos un momento para analizar y limpiar nuestras "gafas optimistas" con las que a veces decidimos ver el mundo. En tal sentido, parece que la vida es, entre otras cosas, una suma de factores que provocan en el ser humano sentimientos de molestia, desazón, e incluso sufrimiento. Ya la experiencia del sufrimiento ha sido históricamente aceptada como parte intrínseca de la vida en múltiples ámbitos del saber humano (Bayés, 1998; Wilson \& Luciano, 2002; Luna, 2008). La vida sería, entonces, amarga e imposibilitante, una promesa incumplida. Sin embargo, es necesario mencionar que se supondrá aquí (al igual que Fernando Savater cuando prologa La conquista de la felicidad de Bertrand Russell) que el lector de estas líneas (y también el cliente con el que trabajaríamos) goza de razonable buena salud, con un trabajo no 
esclavizador que le permite ganarse la vida sin atroces agobios, que vive en un país donde está vigente un régimen político con presumible y aceptable igualdad de derechos y a quien no afecta personalmente ningún accidente fatal. Es decir, lo propuesto será válido para aquellos "privilegiados" que no luchan por su mera supervivencia, que disfrutan de una existencia soportable pero que quisieran que fuese realmente satisfactoria... o para aquellos, aún más frecuentes, empeñados en hacerse insoportable a sí mismos una vida que objetivamente no tendría por qué serlo.

Con estas aclaraciones, es preciso que en este momento se deba poner en claro a qué nos referimos con sufrimiento. Bayés (1998) nos presenta la ya clásica definición de Cassell (1992): "estado de malestar inducido por la amenaza de la pérdida de integridad o desintegración de la persona, con independencia de su causa". Allí mismo refiere la definición de Chapman \& Gravin (1993): "un estado afectivo, cognitivo y negativo complejo caracterizado por la sensación que experimenta la persona de encontrarse amenazada en su integridad, por su sentimiento de impotencia para hacer frente a esta amenaza y por el agotamiento de los recursos personales y psicosociales que le permitirían afrontarla". Estas definiciones permiten observar la asociación entre el sufrimiento y el fenómeno de la percepción de control. Muchas personas sufren por el supuesto origen desconocido de su sufrimiento, o también cuando creen que no pueden aliviarlo o incluso cuando tal sufrimiento podría ser objetivamente insignificante pero la persona, al rechazarlo, lo renueva constantemente volviéndose un ciclo de negación destructiva. En suma, y paradójicamente, las personas sufren -más de lo debido- cuando se empecinan en tratar de controlar su sufrimiento, negando, ignorando o menospreciando la inexorabilidad del sufrimiento humano. Con todo ello, se puede decir que al analizar el sufrimiento, en el sentido de actualizar constantemente las penalidades de la vida tal y como uno las vive y/o poner en el presente el sufrimiento futuro, la entenderemos como una experiencia reservada a los seres humanos verbales como especie que, a través de llamada inteligencia superior, ha conseguido desde hace siglos llegar a manipular las condiciones inmediatas ambientales y biológicas con un alto grado de precisión, pero que a la vez está mostrando, principalmente desde mediados del último siglo, una repercusión de efectos psicológicos insospechados.

Sucede que está actualmente muy arraigado el siguiente precepto: "Bienestar y sentirse bien, igual a normal. Sufrimiento y sentirse mal, igual a anormal". Se entiende así que hay que buscar por todos los medios sentirse bien y evitar el sufrimiento. De allí que sucedan situaciones como estas: "debo sentirme querido pero sin sentirme mal en el proceso o debo tener ideas claras pero sin dudar antes". Parece algo muy extendido que sentirse bien resulta equivalente a dar la espalda a las sensaciones, pensamientos y otros eventos privados, ya que estos se entienden como negativos y anormales sirviendo como barrera para poder vivir. En la misma lógica, sentirse mal y creerse mentalmente sano, no tiene coherencia socialmente. Ni hablar de alguien que diga: "me siento mal, pero estoy bien". Esto será entendido como una contradicción pavorosa. En suma, el sufrimiento se debe negar, evitar o controlar; sin embargo, como mencionan Wilson \& Luciano (2002 p. 33) "cuando los eventos privados vividos negativamente llegan a actuar como barreras o causas que impiden vivir feliz, y la persona solo sabe luchar contra sí misma al hacerlo contra sus eventos privados, y esta lucha deliberada en lugar de eliminar lo que "molesta" 
lo hace cada vez más presente, el resultado final es un contexto de sufrimiento por la limitación que este proceder evitativo engendra (ya que la única estrategia es evitar) y que según los valores de uno puede ser una limitación destructiva".

Un punto importante respecto a lo mencionado es que resulta aparentemente contradictorio que la parte del mundo donde se haya más patentes las disfunciones psicológicas sea precisamente el mundo donde está al alcance la tecnología avanzada, donde aparentemente la mayoría de las penalidades están mitigadas para la gran cantidad de la población. Así, parecería que la lógica de sentirse bien para poder vivir atrofia las capacidades (Wilson \& Luciano, 2002), ya que ponemos como condición necesaria para poder vivir una situación, que en principio, no se cumple a cabalidad. En esa línea, parecería altamente probable que en los lugares en donde el sacrificio y el sufrimiento se consideran como indesligables a la vida, los trastornos psicológicos no cunden. Tal vez esta anotación podría ir en contra a lo referido por las Naciones Unidas al conmemorarse el Día de la Salud Mental: "Los trastornos mentales [...] demasiadas veces se presentan asociados a la pobreza, la marginación y las desventajas sociales" (OMS, 2008). Hay que tener cuidado con esto porque a lo que principalmente se refiere, en función a los datos que maneja la Organización Mundial de la Salud (OMS), es a las enfermedades neurológicas y psiquiátricas que se diferencian notoriamente de los trastornos psicológicos (y ni que decir de los malestares subclínicos y problemas de la vida cotidiana, que es a lo que principalmente nos referimos). No olvidemos que la OMS es una entidad principalmente médica, y así, el concepto de Salud Mental tiene un claro sesgo nosológico.

Parece pues, que la persona está frente a un rechazo de toda forma de incomodidad como si no fueran fenómenos naturales que devienen del simple hecho de ser humanos. Se piensa, equivocadamente, que el bienestar nos es consustancial y el sufrimiento un problema al que debemos erradicar rápida y efectivamente. En la actualidad, se busca un grado de bienestar que siglos atrás solo se hubiera concebido como digno de dioses (un claro ejemplo del incremento desmesurado de las expectativas que más adelante se analizará). La elevada intolerancia hacia el desagrado ha hecho que perdamos la capacidad necesaria para invertir tiempo y esfuerzo en empresas o tareas que pueden aportar beneficios a largo plazo. Esta impaciencia nos lleva a exigir de nuestras acciones la satisfacción de manera inmediata, aquí y ahora. Hoy vivimos, alentados por la sociedad de consumo, en la necesidad de satisfacer nuestros deseos de manera inmediata o por lo menos a cortísimo plazo. Según Ursúa (2005), queremos evitar sea como sea el menor desagrado y disgusto, y esto tiene como consecuencia inmediata el imposibilitar ciertos procedimientos para llegar al placer que estriban precisamente en el contraste y sus efectos. El entramado complejo "desagrad. intolerancia" parece crecer cada vez más en nuestras vidas que buscan con ahínco las situaciones excitantes, novedosas, dando lugar a un "aburrimiento mortal", a una vida aplanada y artificial. Pretender evitar todo sufrimiento significa sustraerse de una parte esencial de la vida misma. 


\section{EL INCREMENTO DE LAS EXPECTATIVAS Y LA IMPOSIBILIDAD DE SATISFACERLAS}

La exageradamente mencionada globalización ha traído consigo una situación no tan mencionada, a saber: la feroz homogenización de los gustos y preferencias. Esto podría no tener mayores inconvenientes sino fuera porque somos muy diferentes en nuestras capacidades de adquisición y satisfacción. La cultura de masas logró hacer llegar las mismas ambiciones para todas las personas. Vivimos en un mundo que homogeniza despiadadamente sin tomarse el tiempo en las diferencias y no hay peor cosa que hacer iguales a los que no lo son. La gran urbanización, por ejemplo, en América Latina desde mediados del siglo pasado, condujo a la ciudad a millones de inmigrantes, cambió sus expectativas, las agigantó e igualó a las del resto de la sociedad. Pero, paradójicamente, la misma sociedad les cerró los caminos para poder satisfacerlas. En América Latina, como señala Briceño-León (2007) nos encontramos con una asimetría entre la homogeneidad en las aspiraciones y la heterogeneidad en la capacidad de colmarlas. Somos terriblemente iguales en lo que deseamos y espantosamente desiguales en nuestras posibilidades reales de lograrlo.

Lo que para los padres y abuelos llegó a ser un sueño accesible, es decir, hospitales cercanos, escuelas, luz eléctrica, una televisión en casa, etc., para los hijos esos logros ya no significan nada. Estos nacieron en un mundo donde se les impuso nuevas, y quizá más superficiales, metas de consumo. El carácter omnímodo de la comunicación de masas ha conseguido que un joven de familia clase media que se prepara para ingresar a la universidad, y uno de familia pobre y desempleado, tengan los mismos gustos y los mismos antojos.

Esta asimetría entre expectativas y logros plantea un drama, pues ya que los caminos prescritos por la sociedad: empleo, esfuerzo y ahorro, no parecen ser suficientes para alcanzar los fines, muchas personas asumen los caminos proscritos, a saber, el uso de la fuerza para alcanzar lo que desea o la frustración y el desequilibrio psicológico por no lograrlo.

\section{LA EXCESIVA NECESIDAD DE CONTROL}

La cultura popular le ha dado un papel fundamental a los eventos privados (por ejemplo: cogniciones y emociones) como factores causantes del comportamiento. Es frecuente que la conducta "anormal" sea explicada, dentro de la comunidad verbal, como el resultado de eventos internos tales como las emociones o los pensamientos. Ello conlleva a que la gente piense que, para controlar su conducta problemática, se debe controlar los eventos internos que la causan. Así, por ejemplo, si una persona aprende de su comunidad verbal que la causa del temor a hablar en público es la ansiedad o los pensamientos negativos, trataría de controlar dicha emoción y dichos pensamientos con el fin de controlar ese temor (Rey, 2004). Este énfasis en lo intrapsíquico ha oscurecido el contexto social, aunque ningún enfoque sensato daría por supuesto aquél sin el concurso de éste (Martín, 2006). Con todo ello, parece ser que estaríamos frente a otra paradoja, la de la exigencia personal de hacerse del control de los eventos privados (supuestamente generadores de malestar psicológico) y el casi nulo control que se tendría sobre las preferencias, 
deseos y expectativas que son el resultado de un proyecto de marketing ajeno al control de los individuos (consumidores). La necesidad de control llevaría aparejada una enorme impaciencia, irritabilidad, miedo al fracaso, baja tolerancia a la frustración y la incapacidad para construir relaciones recíprocas. Podría decirse que las personas -en este caso se trata de una descripción del neurótico moderno- viven como si carecieran de historia; se trata de una postura en la que el sujeto se evade de la responsabilidad de hacerse cargo de su propia existencia, como si no le incumbiera dirigirla (Martín, 2006). Párrafos arriba ya se hizo notar la asociación entre el sufrimiento y el fenómeno de la percepción de control que llevaría a un círculo vicioso dañino. El carácter ubicuo del sufrimiento en los humanos es un hecho, y quedar atrapados en un patrón destructivo de no aceptación del sufrimiento socioverbal es algo cada vez más común.

Con todo lo dicho, permítaseme una digresión: no podemos evitar decir que, ante este escenario, uno de los caminos a seguir es la labor de desarrollo moral de la sociedad en su conjunto (como tarea pendiente e ineludible de todos nosotros); así, pensar que las estrategias de moralización que podamos proponer tomarán en cuenta únicamente a los individuos (aislados) es un error fundamental. Es necesario considerar la posibilidad de que si han de ensayarse distintas o nuevas estrategias de moralización más efectivas, se requiere de por lo menos una revisión de la teoría de la sociedad y de los hombres; una revisión de la estructura y los conceptos éticos, y una revisión de la teoría en que se basan las tecnologías educacionales y de atención de salud que se aplican en el mundo (Ortiz, 2007); sin embargo, esa es una historia mayor y lo que presentaremos aquí (sin ir en contra de lo antedicho) son las posibilidades que propone la Terapia de Aceptación y Compromiso (TAC) para vivir una vida más humana a pesar de estas circunstancias.

\section{TERAPIA DE ACEPTACIÓN Y COMPROMISO (TAC²)}

Se considera que en la actualidad estamos en la "tercera generación de terapias conductuales" (Pére.Álvarez, 2006), siendo la primera, aquellas que devenían directamente de los dos paradigmas básicos (condicionamiento clásico y operante). Este tipo de terapias tenían una marcada vocación contextualista y centrada en el análisis del caso individual. La segunda generación es la que podría denominarse en general "cognitiv.conductual", que asume fuertes influencias del modelo de procesamiento de información, por un lado, y del modelo médico, por el otro, teniendo una inclinación internalista, nosológica y con clara tendencia a estandarizar los casos. En esa línea, otorgan un papel primordial a los eventos cognitivos (y también emotivos) como causantes mecánicos del comportamiento, llevando como conclusión la importancia que le otorgan al control de dichos eventos en el tratamiento psicológico. Así, llegamos a la tercera generación, mencionando que su principal aporte está en el replanteamiento contextual como forma de desmedicalización de los trastornos psicológicos, con la formulación, en el análisis del comportamiento, de una nueva y más adecuada categoría de orden funcional (trastorno de evitación

2 Kohlenberg, Tsai, Ferro, Aguayo, Fernández, \& Virúes (2005) refieren que es incierto el uso del acrónimo en inglés (ACT) 0 castellano (TAC). Dado que la versión inglesa se encuentra ampliamente difundida en textos castellanos y tiene una funcionalidad semántica en el contexto de la terapia (por ejemplo, actuar), mientras que el acrónimo en castellano puede conducir a confusiones con otros conceptos (por ejemplo, tomografía axial computarizada). De allí que prefieran usar la versión inglesa; sin embargo, aquí usaremos la versión en castellano con la aclaración del caso. 
experiencial, como alternativa la psicopatología actual) y, en función al tratamiento, con el abandono de la lucha contra las síntomas y en su lugar la reorientación de la vida.

Una de las principales representantes de esta tercera generación es la Terapia de Aceptación y Compromiso (TAC) propuesta por Hayes, Stroshal \& Wilson (1999), y afianzada en idioma castellano con la publicación de Wilson \& Luciano (2002). Proviene del análisis funcional del comportamiento verbal de tradición skinneriana, que se sigue desarrollando y que es objeto de exposición y análisis continuamente. Se trata de una forma de psicoterapia experiencial conductual y cognitiva basada en la teoría de los marcos relacionales del lenguaje y la cognición humana (para una aproximación a la teoría de los marcos relacionales véase Hayes, Barne.Holmes \& Roche, 2001; Wilson \& Luciano, 2002; Barne.Holmes, Rodríguez \& Whelan, 2005; Luciano \& Valdivia, 2006; Gómez, López \& Mesa, 2007).

La TAC trabaja entre la aceptación y el cambio. Entre aceptar lo que no se puede cambiar y comprometerse a cambiar lo que se puede cambiar y merece la pena para el cliente. $\mathrm{Al}$ encuadrarse dentro del conductismo radical, considera que la cognición es conducta $y$, como tal, sujeta a las mismas leyes que cualquier otro comportamiento. De forma que lo importante para dicha terapia no es el contenido de los pensamientos, sino la función que tienen en el contexto en el que se dan. Por eso, apenas emplea la reestructuración cognitiva, porque su intención principal no se dirige a cambiar el contenido de los pensamientos, sino que modifica la función que tienen (García, 2007).

\section{Evitación experiencial}

Entre los avances de la tercera generación, la TAC trabaja con la llamada evitación experiencial, que puede definirse como un fenómeno que ocurre cuando una persona no está dispuesta a ponerse en contacto con experiencias privadas particulares (por ejemplo: sensaciones corporales, emociones, pensamientos, recuerdos, predisposiciones conductuales) e intenta alterar la forma o la frecuencia de esos eventos y el contexto que los ocasiona (que en algunos casos se trata de un seguimiento de reglas verbales relativas al control estricto de emociones y cogniciones). La evitación experiencial es planteada como una dimensión funcional que podría integrar una variedad de diagnósticos del sistema DSM, por ejemplo, todos los trastornos de ansiedad (trastorno obsesiv.compulsivos, la agorafobia con ataques de pánico, el estrés pos.traumático), el abuso de sustancias, en la anorexia y la bulimia, los trastornos del control de impulsos, los síntomas psicóticos, el afrontamiento de enfermedades, los procesos en los que el dolor juega un papel esencial e incluso, los trastornos de personalidad límite, todos ellos podrían ser clasificados como trastornos de evitación experiencial (Ferro, 2000 \& Kohlenberg et al., 2005; Luciano \& Valdivia, 2006). Así pues, la evitación experiencial es un proceso patogénico que se ofrece como una alternativa funcional a los sistemas tradicionales de clasificación basados en la topografía.

De igual modo, la regulación de la evitación experiencial que el paciente lleva a consulta es tratada en las terapias de segunda generación -incluidas las farmacológicas-, siguiendo la lógica de tratar de reducir directamente el malestar y cualquier otro evento privado con 
esas características (por ejemplo, cambiar los pensamientos irracionales por racionales, reducir el temor a lo que fuera, la tristeza, el desaliento, los recuerdos y sensaciones de malestar, las voces, elevar la autoestima, etc.). En esa línea, la aproximación terapéutica de la TAC al trastorno de evitación experiencial, centrada en el análisis de los contextos verbales que sustentan la evitación experiencial destructiva, es radicalmente diferente (Luciano \& Valdivia, 2006).

\section{Contextos verbales de evitación experiencial}

Desde la teoría de los marcos relacionales, habría varios contextos verbales definiendo el trastorno de evitación experiencial:

1. Contexto verbal de significado literal. El contexto de la "literalidad" es un producto ineludible del comportamiento verbal y supone responder a un evento en términos de otro, esto es lo que permite la actuación simbólica en tanto que las funciones del referente están parcialmente presentes en el símbolo y viceversa (Wilson \& Luciano, 2002). Cuando la distinción entre las palabras y sus referentes se pierde y ambos llegan a ser funcionalmente equivalentes, las palabras pueden terminar siendo evitadas tanto como los eventos reales con los que se relacionan. No quedar atrapado en la literalidad de las palabras supone aprender a discriminar cuándo la literalidad es útil y cuándo no. Por ejemplo, que la palabra "¡Peligro!" contenga función aversiva y la persona se comporte de acuerdo con ello, será efectivo si lo hace en relación a no fumar (cáncer), no tocar un cable de alta tensión o ante la presencia de una animal peligroso. Pero no lo será cuando "¡Peligro!" se asocie a tener dudas sobre uno mismo, y esto se vive opuesto a la vida, de forma que se lucha contra las dudas para evitarlas tanto como se evitaría el peligro del cable o del animal peligroso.

En tal sentido, Montgomery (2009) reflexiona, respecto al incremento acelerado de las expectativas de consumo material, si en semejante situación no juega un papel nacionalmente idiosincrásico cierto tipo de evitación experiencial. Mucha gente no vive ni disfruta el momento de creciente prosperidad relativa porque se ha acostumbrado a pensar comparativamente siempre en futuro (donde esperaría "tener más") y en presente con respecto a otros mejor ubicados en la escala social. Así, sus relaciones verbales se ajustan a un modelo de queja constante sobre "el lado malo" de las cosas (lo que en TAC se llama precisamente "contexto de literalidad"), distrayendo su atención de lo constructivo con el consecuente perjuicio psico(pato)lógico. Esa es una forma de evitación o escape de su responsabilidad en la construcción de su porvenir y del porvenir de su comunidad.

2. Contexto verbal valoración o evaluación. Es la tendencia a evaluar casi todo, y debido a la literalidad, a no distinguir entre las propiedades intrínsecas de un evento ("estoy triste") y sus propiedades arbitrarias establecidas socialmente ("estar triste es malo"). Implica la dificultad para diferenciar las dimensiones del yo, construidas socialmente en el desarrollo, de modo que, sin diferenciar el yo que sirve de contexto a todos los pensamientos, sólo se actúa fusionado a las propiedades verbales de dichos pensamientos (Luciano \& Valdivia, 2006). De ahí que un "problema psicológico" pueda originarse por la valoración social que tiene la conducta en cuestión. Por 
ejemplo, una pareja de esposos podría consultar por sus prácticas sexuales, a pesar de que éstas son muy satisfactorias para ellos, ya que su grupo social las califica como "aberrantes" (Rey, 2004).

3. Contexto verbal de la explicación. Consistente en dar razones y el apoyo cultural para la emoción y la cognición como causantes de la conducta. Viene potenciado por el punto de vista cultural de que el comportamiento se (mal)explica por las emociones y los pensamientos (por ejemplo, "estos pensamientos son terribles y no puedo vivir con ellos, no puedo trabajar, no puedo estar con mis hijos... Tengo que quitarlos, etc."). Rey (2004) nos dice que la comunidad verbal ofrece explicaciones para los eventos del mundo real, incluyendo la conducta de la gente. Así, es común que dicha comunidad explique la conducta anormal con base en explicaciones mentalistas. Por ejemplo, los accesos de tristeza repetidos se explican porque la persona "es depresiva". Aunque las palabra "depresiva" sea solamente un rótulo verbal que la comunidad brinda a unas experiencias privadas, la misma comunidad da a entender de esa manera que estas experiencias son provocadas por estados subjetivos subyacentes.

4. Contexto verbal del control de la emoción y la cognición. Este contexto es la clave que da sentido a los anteriores en tanto que es un actuar siguiendo esas razones paradójicas, por ejemplo, comportarse antagónicamente a pensamientos molestos, tomados como causas: "si pudiera quitármelos estaría bien, sería otra persona y podría hacer muchas cosas". Así, sólo si esas "causas" desaparecen, la persona estaría en disposición de "darse permiso" para actuar en dirección a aspectos valiosos de su vida. Este último contexto es el que cierra el círculo contingencial al proporcionar el potente reforzador de tener razón (al seguir las reglas para poder vivir) que acompaña al alivio inmediato, aunque efímero. Y todo ello a pesar del costo a largo plazo de tales estrategias (más malestar y menos acciones al servicio del reforzamiento positivo) (Luciano \& Valdivia, 2006). Controlar nuestros eventos privados se ha vuelto una exigencia personal muy costosa.

\section{Componentes o fases de la TAC}

Tal como se dijo anteriormente, el proceso de aceptación y cambio no implica resignarse a lo negativo, sino actuar de acuerdo con una dirección elegida por el mismo usuario, coherente con sus metas y valores. Es decir, las fases o componentes son tomados en el sentido de metas que buscan primordialmente generar un estado de distanciamiento comprensivo hacia los problemas, con el fin de ayudar al usuario a aceptarlos y a comprometerse a elegir una opción de vida, a pesar de los mismos (Pérez-Álvarez, 1996, citado por Rey, 2004).

Tales componentes o fases de la TAC se presentan con ligeras variaciones en diferentes autores, pero manteniendo las bases originales. En este caso se delimitarán seis fases en el desarrollo de esta terapia, cada una tendría sus objetivos, estrategias e intervenciones propias (Wilson \& Luciano, 2002; Kohlenberg et al., 2005; Rey, 2004).

La fase uno es la denominada desesperanza creativa, que pretende romper los repertorios de evitación que el cliente lleva tiempo aplicando para solucionar el problema y que 
constituyen parte del mantenimiento de ese mismo problema; también se pretende en esta fase eliminar el soporte verbal y social que mantiene esa evitación continua. La fase dos, que podría denominarse "el control es el problema y no la solución", que pretende demostrarle al individuo que sus intentos de control son el propio problema y donde el terapeuta ha de moldear esta aproximación a su problema, para que deje activamente de hacer lo que hacía hasta ese momento. La fase tres, llamada construyendo la aceptación, trata de desmantelar el lenguaje, las funciones del lenguaje y su relación con las emociones o los recuerdos del cliente; según los autores, es un proceso de "desliteralización" del propio lenguaje y de tratar de proponer como alternativa a la evitación la aceptación psicológica. En la fase cuatro, se establece el Yo como contexto y no como contenido; se trata de situar verbalmente la construcción del yo como persona, como centro desde el que actuar y diferenciarlo de las emociones, pensamientos o recuerdos. En la fase cinco, denominada valorando, se trata de analizar los valores del cliente, los fundamentos desde los que actúa y también las bases verbales sobre las que se asientan esos valores y, a partir de ello, escoger y valorar una dirección propia. La fase final es denominada estar dispuesto al compromiso; con ella se pretende comprometer al individuo con el cambio de su conducta, no a través de instrucciones directas, sino valorando las conclusiones y decisiones a las que llega el cliente al final de la terapia, en la cual ya está dispuesto a adoptar cambios por sí mismo.

La TAC ha sido discutida, tal vez por aparentar ser una intervención hasta cierto punto "manualizada"; sin embargo, y por citar a dos fuentes importantes, en Kohlenberg et al. (2005) y Luciano \& Valdivia (2006) se muestra la pluralidad de entidades diagnósticas en los que se aplica eficazmente la terapia. De igual modo, las razones por las cuales se presenta a la TAC como uno de los modelos psicoterapéuticos más pertinentes, en función al análisis esbozado de la realidad problemática generadora de una multiplicidad de malestares psicológicos, es porque esta terapia aporta a la psicología clínica entre otras cosas: una filosofía de vida, una perspectiva cultural sobre la que entender y tratar dichos trastornos psicológicos y una alternativa psicopatológica en el denominado trastorno de evitación experiencial. Asimismo, es muy importante precisar que rompe con la tradición terapéutica existente, en el sentido de hacer un análisis no sintomático de los problemas psicológicos, sino funcional; y porque introduce una forma conductual de analizar las emociones, sentimientos, recuerdos y los pensamientos de las personas.

\section{CONCLUSIONES}

Estamos en una época en la que ser feliz se ha vuelto un deber inflexible. Ya lo dijo el filósofo español Gustavo Bueno en una entrevista sobre la publicación de su libro El mito de la felicidad: "Ahora ser feliz es un deber; y el que no quiere ser feliz es un degenerado a los ojos de la sociedad" (Espiniella, 2005). Por desgracia, no podemos dejar de decir que los psicólogos tenemos algo que ver en esta confusión, ya sea porque avalamos esta inclinación actual en nuestra quehacer profesional o porque sencillamente no nos parece un situación problemática. Sumado a ello está el inmenso mercado de la "literatura de autoayuda" de consumo masivo, que lo que ha hecho es reafirmar la tendencia muy actual de entender la felicidad como un bien al alcance de todos. Esta 
democratización despiadada ha traído consigo, por un lado, la espantosa moda de "Las 10 claves para...", y por el otro, el ascenso y exaltación de los "gurúes emocionales", especialistas en mezclas cuas.mágicas y sin un orden claro, de "técnicas" de diferentes procedencias: mentales, espirituales, energéticas, sensoriales, religiosas, etc., y que, por lo visto, tienen un gran éxito.

En este sentido, y contrariamente a la suposición de los que reprueban el conductismo, este enfoque, y principalmente la TAC, no incurren en las acostumbradas promesas tan propias de la modernidad de que todo tiene solución práctica. Pero tampoco tiene nada que ver con "revolcarse" en el sufrimiento o contar de manera indolente "las hojas de las margaritas", sino con vivir una vida humana más comprometida y vital. Así, hay que tener muy presente que el análisis de la mayoría de los malestares psicológicos contemporáneos revela una abismal desproporción entre lo que creemos que debemos ser, sentir y poseer y lo que realmente podemos ser, sentir y poseer. Es por esto que nos alineamos a la sensatez que representa el aceptar lo que no se puede cambiar y comprometerse a cambiar lo importante para el cliente.

En palabras de Marino Pérez Álvarez (2002, p. 20), "muchos podrán decir que el conductismo que les enseñaron no era este. $Y$ es que a mucha gente le enseñaron mal el conductismo. El psicólogo ya hecho estará a tiempo para remediar sus prejuicios y, por su parte, el estudiante lo estará para empezar bien".

\section{REFERENCIAS BIBLIOGRÁFICAS}

1. Barne.Holmes, D.; Rodríguez, M. \& Whelan, R. (2005). La teoría de los Marcos Relacionales y el análisis experimental del lenguaje y la cognición. En: Revista Latinoamericana de Psicología, 37(2), pp. 255-275.

2. Bautista, J. \& Quiroga, E. (2005). La relevancia de un planteamiento cultural de los trastornos de la personalidad. En: Psicothema, 17(3), pp. 422-429.

3. Bayés, R. (1998). Psicología del sufrimiento y de la muerte. En: Anuario de psicología, 29(4), pp. 5-17.

4. Briceñ.León, R. (2007). Violencia urbana en América latina: Un modelo sociológico de explicación. En: Espacio abierto, 16(3), 541-574.

5. Espiniella, R. (2005). "Don’t worry, be happy!" En: El Comercio. 4 de mayo de 2005. (Gijó.España). Recuperado desde http://www.fgbueno.es/hem/2005f04.htm

6. Ferro, R. (2000). Aplicación de la terapia de aceptación y compromiso en un ejemplo de evitación experiencial. En: Psicothema, 12(3), pp. 445-450.

7. García, J. (2007). La terapia de aceptación y compromiso: tercera generación de la terapia cognitivo conductual. Recuperado desde http://www.psicoterapeutas.com/ pacientes/act/brevepresenact.htm

8. Gómez, S.; López, F. \& Mesa, H. (2007). Teoría de los marcos relacionales: algunas implicaciones para la psicopatología y la psicoterapia. International Journal of Clinical and Health Psychology, 7(2), pp. 491-507. 
9. Hayes, S.C.; Strosahl, K.D. \& Wilson, K.G. (1999). Acceptance and commitment therapy. Nueva York: The Guilford Press.

10. Hayes, S.C.; Barne.Holmes, D. \& Roche, B. (2001). Relational frame theory: A pos.skinnerian account of human language and cognition. Nueva York: Kluwer Academic/Plenum Publishers. Capítulo 8 traducido al castellano, disponible en http://www.abacolombia.org.co/bv/semskinner/rtf_precis.pdf.

11. Kohlenberg, R.; Tsai, M.; Ferro, R.; Aguayo, L.; Fernández, A. \& Virúes, J. (2005). Psicoterapia analític.funcional y Terapia de aceptación y compromiso: teoría, aplicaciones y continuidad con el análisis del comportamiento. En: International Journal of Clinical and Health Psychology, 5(2), pp. 349-371.

12. Luciano, C. M. \& Valdivia, M. (2006). La terapia de aceptación y compromiso (TAC). Fundamentos, características y evidencia. En: Papeles del psicólogo, 27(2), pp. 79-91.

13. Luna, R. (2008). Cambio social y cultura de la resignación y el sufrimiento. Espacio abierto, 17(2), pp. 267-284.

14. Martín, F. (2006). Cambios sociales y trastornos de la personalidad posmoderna. Papeles del Psicólogo, 27(2), pp. 104-115.

15. Montgomery, W. (2009). Mindfulness y gaudibilidad: Categorías en terapia de conducta para tratar la evitación experiencial. En: Revista de Psicología de la Universidad Inca Garcilaso de la Vega, 1(1), pp. 55-61.

16. Organización Mundial de la Salud (OMS) (2008). Mensaje sobre el Día Mundial de la Salud Mental del Secretario General. Recuperado desde http://www.who.int/ mental_health/mhgap/UN_speech_mhgap_spanish.pdf

17. Ortiz, P. (2007). Ética social. Lima: UNMSM.

18. Pére.Álvarez, M. (2002). Prefacio. En: Wilson, K. G. \& Luciano, C. M. Terapia de aceptación y compromiso. Un tratamiento conductual orientado a los valores, pp. 19-20. Madrid: Pirámide.

19. Pére.Álvarez, M. (2006). La terapia de conducta de tercera generación. En: EduPsykhé: Revista de psicología y psicopedagogía, 5(2), 159-172.

20. Rey, C. (2004). La terapia de aceptación y compromiso (ACT): Sus aplicaciones y principales fundamentos conceptuales, teóricos y metodológicos. En: Suma Psicológica, 11(2), pp. 267-284.

21. Ursúa, N. (2005). Los ocho pecados mortales de la humanidad civilizada. Una relectura de Konrad Lorenz y los problemas de la naturaleza humana. En: Ludus Vitalis, XIII(24), pp. 165-180.

22. Wilson, K.G. \& Luciano, C. M. (2002). Terapia de aceptación y compromiso. Un tratamiento conductual orientado a los valores. Madrid: Pirámide. 